\title{
A Sylvester Theorem for Conic Sections
}

\author{
James A. Wiseman and Paul R. Wilson \\ Rochester Institute of Technology, Department of Mathematics, Rochester, NY 14623, USA
}

\begin{abstract}
If $\mathbf{S}$ is a finite set of points in the plane and no conic contains all points of $\mathbf{S}$, then $\mathbf{S}$ determines a conic which contains exactly five points of $S$.
\end{abstract}

In 1893 Sylvester [13] posed the following problem: given a set of $n$ points in the Euclidean or projective plane, not all collinear, is there necessarily a line (a Sylvester line) which contains exactly two of the points? Sylvester offered no solution and the problem was not solved until, in 1933, the first proof that a Sylvester line must exist was given by Gallai. In 1943 Erdös publicized Gallai's solution by posing the Sylvester question as a problem in the American Mathematical Monthly [5]. One response to Erdös's problem, by Steinberg [12], gave the first completely projective proof of Sylvester's conjecture. Another important proof of the conjecture, using metric techniques, was given by Kelly in 1948 [9].

In 1951 Motzkin [11] showed that among the planes determined by a finite set of points in 3-space there must be at least one, which he called "ordinary," with the property that all but one of the points of the original set which lie on that plane are collinear.

Hansen [6], in 1965, generalizing methods first introduced by Steinberg, proved an $n$-dimensional analogue to Motzkin's results: among the $(k-1)$-flats determined by a finite set of points in a $k$-dimensional space there must be at least one (an ordinary flat) with the property that all but one of the points of the original set which lie in that $(k-1)$-flat lie in a $(k-2)$-flat.

In 1983 Borwein [2] used unimodal Haar vector spaces to prove a different generalization of Sylvester's theorem: if $E$ is a finite set of points in the plane such that no two points of $E$ lie on the same vertical line and no polynomial of degree less than $n+1$ contains all points of $E$, then for $k=1, \ldots, n$ there is a polynomial of degree $k$ containing exactly $k+1$ points of $E$. Borwein's proof echoes the proof that Kelly gave for Sylvester's theorem; his result may be deduced using the Motzkin-Hansen theorem and the methods of the present paper. 
We add to the Sylvester-Gallai theory by proving a Sylvester type theorem for conic sections.

Definition 1. A subset $\mathbf{S}$ of the Euclidean plane is said to be of order $n$ if there is a polynomial $p(X, Y) \in \mathbb{R}[X, Y]$ of degree $n$ such that every point of $S$ is a zero of $p$. A subset of the plane is called an $n$ th-order algebraic curve if it consists exactly of the zeros of an $n$ th-degree polynomial $p(X, Y)$.

Definition 2. Let $\mathbf{S}$ be a subset of $\mathbb{R}^{2}$ and $\mathbf{C}$ be an $n$ th-order curve in the plane. We say that $\mathbf{C}$ is determined by $\mathbf{S}$ if, for any $n$ th-order curve $\mathbf{C}^{\prime}$,

$$
\mathbf{C}^{\prime} \cap \mathbf{S} \supseteq \mathbf{C} \cap \mathbf{S} \Rightarrow \mathbf{C}^{\prime}=\mathbf{C} .
$$

That is, $\mathbf{C}$ is determined by $\mathbf{S}$ if it contains certain points of $\mathbf{S}$ and is the only $n$ th-order curve to contain those points.

Definition 3. Let $\mathbf{S}$ be a subset of $\mathbb{R}^{2}$ and $\mathbf{C}$ be an $n$ th-order curve determined by $\mathbf{S}$. We call $\mathbf{C} n$-elementary if $|\mathbf{C} \cap \mathbf{S}|=n(n+3) / 2$; $\mathbf{C}$ is called ordinary if there is a point $x \in \mathbf{S}$ such that $\mathbf{C}$ is not determined by $\mathbf{S}-\{x\}$. If $\mathbf{S}$ determines an $n$-elementary curve we will say that $\mathbf{S}$ is $n$-elementary. If $\mathbf{S}$ determines an ordinary $n$ th-order curve we will say that $\mathbf{S}$ is $n$-ordinary.

For example, let $\mathbf{S}=\{A, B, C, D, E\}$ as shown in Fig. $1 . \mathbf{S}$ is a second-order set which is 1-elementary, it determines five first-order curves, only one of which, $B C D E$, is not elementary. $S$ is also 1-ordinary; of the five lines it determines only $B C D E$ is not ordinary.

Definition 4. Let $\mathbf{T} \subseteq \mathbb{R}^{n}$. We use $\langle\mathbf{T}\rangle$ to indicate the smallest affine subspace of $\mathbb{R}^{n}$ containing $\mathbf{T}$. We write $\operatorname{dim}\langle\mathbf{T}\rangle$ for the dimension of $\langle\mathbf{T}\rangle$ and will sometimes write $\operatorname{dim} \mathbf{T}$. We call $\langle\mathbf{T}\rangle$ a $k$-flat if $\langle\mathbf{T}\rangle$ is a $k$-dimensional affine subspace of $\mathbb{R}^{n}$. An $(n-1)$-fiat will be called a hyperplane.

Definition 5. Let $\mathbf{T} \subseteq \mathbb{R}^{n}$ have dimension $n$. A hyperplane $\mathbb{H}$ in $\mathbb{R}^{n}$ is said to be determined by $\mathbf{T}$ if for any hyperplane $\mathbb{H}^{\prime}$

$$
\mathbb{W}^{\prime} \cap \mathbf{T} \supseteq \mathbb{H} \cap \mathbf{T} \Rightarrow \mathbb{H}^{\prime}=\mathbb{H} \text {. }
$$

A hyperplane, $\mathbb{H}$, determined by $T$ is called elementary if $|\mathbb{H} \cap T|=n$; a hyperplane, $\mathbb{H}$, determined by $\mathbf{T}$ is called ordinary if there is an $x \in \mathbf{T}$ such that $\operatorname{dim}\langle\mathbb{H} \cap$ $(\mathbf{T}-\{x\})\rangle=n-2$. (In words: $H$ is ordinary if all the points of $H \cap T$, save one, lie in an ( $n-2)$-flat.) We call $\mathbf{T}$ elementary (resp. ordinary) if it determines at least one elementary (resp. ordinary) hyperplane.

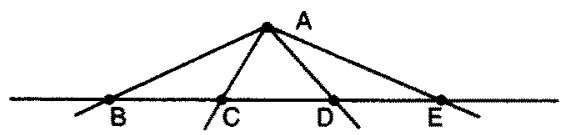

Fig. 1 
We note that, in terms of these definitions, Sylvester's theorem says: if $\mathbf{S} \subseteq \mathbb{R}^{2}$ is not first order, then it is 1-elementary.

We omit the easy proof of the following result.

Corollary. Let $\mathrm{T} \subseteq \mathbb{R}^{n}$ and $\mathbb{H}$ be a hyperplane in $\mathbb{R}^{n}$. Then $\mathrm{H}$ is determined by $\mathbf{T}$ iff $\operatorname{dim}(\mathbf{H} \cap \mathbf{T}\rangle=n-1$.

Our goal is to prove the following theorem.

Theorem. If $\mathbf{S} \subseteq \mathbb{R}^{2}$ is not co-conic, then there is a conic determined by $\mathbf{S}$ which contains exactly five points of $\mathbf{S}$.

This is Sylvester's theorem extended to conic sections. Put differently, it says that if $S \subseteq \mathbb{R}^{2}$ is not second order, then it is 2-elementary.

As a first step toward the proof we define $T: \mathbb{R}^{2} \rightarrow \mathbb{R}^{5}$ by

$$
T(x, y)=\left(x^{2}, x y, y^{2}, x, y\right) \text {. }
$$

The last two coordinates guarantee that $T$ is $1-1$. The following lemmas establish some of the properties of the map $T$.

Lemma 1. If $\mathbf{S} \subseteq \mathbb{R}^{2}$ is not second order, then $\operatorname{dim} T(\mathbf{S})=5$.

Proof. Suppose not. Then there is a hyperplane $\mathbb{H}$ in $\mathbb{R}^{5}$ such that $T(\mathbf{S}) \subseteq \mathbb{H}$. Let $\mathbb{H}$ have equation

$$
a_{0}+a_{1} x_{1}+a_{2} x_{2}+a_{3} x_{3}+a_{4} x_{4}+a_{5} x_{5}=0
$$

Then, by definition of $T$,

$$
a_{0}+a_{1} x^{2}+a_{2} x y+a_{3} y^{2}+a_{4} x+a_{5} y=0
$$

for all $(x, y) \in \mathbf{S}$; that is, all points of $\mathbf{S}$ lie on a conic. The contradiction proves the lemma.

Lemma 2. A set $\mathbf{S} \subseteq \mathbb{R}^{2}$ determines exactly one conic $\mathbf{C}$ iff $\operatorname{dim} T(\mathbf{S})=4$. In this case $\mathbf{S} \subseteq \mathbf{C}$.

Proof. Suppose S determines a unique conic C. (It follows from Bezout's theorem that any set of five points, no four of which are collinear, determines exactly one conic; see, for instance, [8, p. 54].) Let $\mathbf{C} \cap \mathbf{S}=\mathbf{S}_{1}$. If $\mathbf{S}_{1} \subset \mathbf{S}$ choose a point $X \in S-S_{1}$. Some set of five points in $S_{1}$ determines $C$; by replacing one of those five points with $X$ we determine a new conic. But this contradicts the assumption that $\mathbf{S}$ determines only one conic. It follows that no such point $X$ can exist and $\mathbf{S}=\mathbf{S}_{1}$; i.e., $\mathbf{S} \subseteq \mathbf{C}$. Since $T(\mathbf{C})$ determines a hyperplane $\mathbb{H}_{1}$ in $\mathbb{R}^{5}$ it follows that $\operatorname{dim} T(\mathbf{S}) \leq 4$. If $\operatorname{dim} T(\mathrm{~S})<4$ then there is a hyperplane different from the one containing $T(\mathbf{C})$, say

$$
\mathrm{H}_{2}: \quad b_{0}+b_{1} x_{1}+b_{2} x_{2}+b_{3} x_{3}+b_{4} x_{4}+b_{5} x_{5}=0,
$$


such that $T(\mathbf{S}) \subseteq \mathbb{H}_{2}$. But then all points of $\mathbf{S}$ satisfy

$$
b_{0}+b_{1} x^{2}+b_{2} x y+b_{3} y^{2}+b_{4} x+b_{5} y=0 \text {, }
$$

a conic different from $\mathbf{C}$. Contradiction.

Conversely, suppose $\operatorname{dim} T(S)=4$. Let $\langle T(S)\rangle$ be the hyperplane

$$
H: \quad a_{0}+a_{1} x_{1}+a_{2} x_{2}+a_{3} x_{3}+a_{4} x_{4}+a_{5} x_{5}=0 .
$$

Then $\mathbf{S}$ is contained in the conic

$$
\text { C: } \quad a_{0}+a_{1} x^{2}+a_{2} x y+a_{3} y^{2}+a_{4} x+a_{5} y=0
$$

so $\mathbf{C}$ is the (unique) conic determined by $\mathbf{S}$.

Lemma 3. No three points of $T\left(\mathbb{R}^{2}\right)$ are collinear.

Proof. Suppose $X_{1}, X_{2}, X_{3} \in \mathbb{R}^{2}$ and $T\left(X_{1}\right), T\left(X_{2}\right), T\left(X_{3}\right)$ are collinear in $\mathbb{R}^{5}$. Then $\operatorname{dim}\left\langle T\left(X_{1}\right), T\left(X_{2}\right), T\left(X_{3}\right)\right\rangle=1$. Pick two more points, $X_{4}, X_{5} \in \mathbb{R}^{2}$ so that $\mathrm{S}=\left\{X_{1}, X_{2}, X_{3}, X_{4}, X_{5}\right\}$ determines a unique conic. We have added only two points to a set of dimension 1 , so $\operatorname{dim} T(S) \leq 3$. This contradicts Lemma 2.

Lemma 4. If $\mathbf{S} \subseteq \mathbb{R}^{2}$ has three or more points and its points are collinear, then $\operatorname{dim} T(\mathbf{S})=2$. (In words, the image of $\mathbf{S}$ lies in a plane of $\mathbb{R}^{5}$, but not in a line.)

Proof. By Lemma $3 \operatorname{dim} T(\mathbf{S}) \geq 2$. Let $a$ be the line containing $\mathbf{S}$ and let $P$ and $Q$ be points not on $a$. Then $\mathbf{S}_{1}=\mathbf{S} \cup\{P, Q\}$ determines a unique conic so, by Lemma $2, \operatorname{dim} T\left(\mathbf{S}_{1}\right)=4$; therefore $\operatorname{dim} T(\mathbf{S})$ is 2,3 , or 4 . Since $\mathbf{S}$ does not determine a unique conic, $\operatorname{dim} T(\mathbf{S}) \neq 4$. If $\operatorname{dim} T(\mathbf{S})=3$, then we can find a point $X \in \mathbb{R}^{2}$ such that $\operatorname{dim} T(S \cup\{X\}=4$; but that would imply that $S \cup\{X\}$ determined a conic, a contradiction, for a line and a point do not determine a conic. Therefore $\operatorname{dim} T(\mathbf{S})=2$

Note. Lemma 4 shows that the image under $T$ of a line in $\mathbb{R}^{2}$ is a set of coplanar points in $\mathbb{R}^{5}$. We can prove the result algebraically in the following way.

Let $a=\left\{(x, y) \mid(x, y)=\left(x_{0}, y_{0}\right)+t\left(u_{0}, v_{0}\right), t \in \mathbb{R}\right\}$. Then for $(x, y) \in a$ we have

$$
T(x, y)=T\left(x_{0}, y_{0}\right)+t\left(2 x_{0} u_{0}, x_{0} v_{0}+y_{0} u_{0}, 2 y_{0} v_{0}, u_{0}, v_{0}\right)+t^{2}\left(u_{0}, u_{0} v_{0}, v_{0}, 0,0\right),
$$

i.e., $T(a)=\left\{z \mid z=T\left(x_{0}, y_{0}\right)+t V_{1}+t^{2} V_{2}\right\}$ for points $V_{1}$ and $V_{2}$ in $\mathbb{R}^{5}$. Clearly, the set $T(a)$ is a coplanar, but not collinear.

Lemma 5. If $\mathbf{S} \subseteq \mathbb{R}^{2}$ consists of three noncollinear points, then $\operatorname{dim} T(\mathbf{S})=2$.

Proof. By Lemma 3 the dimension is at least 2, but three points cannot span more than a plane, so the dimension must be exactly 2 . 
Lemma 6. If $\mathbf{S} \subseteq \mathbb{R}^{2}$ consists of four points, not all collinear, then $\operatorname{dim} T(\mathbf{S})=3$.

Proof. Four points cannot span more than three dimensions so the dimension is $\leq 3$. Since the points are not all collinear we can choose one more point $X \in \mathbb{R}^{2}$ such that $\mathbf{S} \cup\{X\}$ determines a unique conic. By Lemma $2 \operatorname{dim} T(\mathbf{S} \cup\{X\})=4$. Thus $\operatorname{dim} T(\mathbf{S})$ is at least 3 , so it must be exactly 3 .

Lemma 7. Let $\mathbf{S} \subseteq \mathbb{R}^{2}$ be a set which is not of order 2, that is, not co-conic. If any line in $\mathbb{R}^{2}$ contains exactly three points of $\mathbf{S}$, then there is a conic which contains exactly five points of $\mathbf{S}$ and which is determined by those five points (i.e, $\mathbf{S}$ is 2-elementary).

Proof. Let $a$ be a line in $\mathbb{R}^{2}$ such that $a \cap \mathbf{S}=\left\{P_{1}, P_{2}, P_{3}\right\}$. Let $\mathbf{S}_{1}=\mathbf{S}-\left\{P_{1}, P_{2}, P_{3}\right\}$. If $S_{1}$ is a collinear set then $\mathbf{S}$ lies in two lines (or one) and is co-conic. This contradiction shows that $S_{1}$ is not collinear, and so must have a Sylvester line $a_{1}$ (a line which contains exactly two points of $S_{1}$ ). Let $P_{4}$ and $P_{5}$ be the points of $\mathrm{S}_{1}$ lying in $a_{1}$. The five points $P_{1}, P_{2}, P_{3}, P_{4}, P_{5}$ determine a conic (consisting of lines $a$ and $a_{1}$ ) which contains no other points of $S$.

Lemma 8. If $\mathbf{S} \subseteq \mathbb{R}^{2}$ has $k \geq 5$ points and $\operatorname{dim} T(\mathbf{S})=3$, then $\mathbf{S}$ consists of $k-1$ collinear points and another point not on the line determined by the first $k-1$ points.

Proof. Consider a set of four points, no three collinear. Note that no matter where a fifth point is added the resulting set of five will determine a unique conic. If $\mathbf{S}$ contained a subset, $\mathbf{S}_{1}$, of four such points, then for any fifth point $X \in \mathbf{S}$ we would have the contradiction

$$
3=\operatorname{dim}\langle T(\mathbf{S})\rangle \geq \operatorname{dim}\left\langle T\left(\mathbf{S}_{1} \cup\{X\}\right)\right\rangle=4
$$

Therefore $\mathbf{S}$ has the property that among any four of its elements at least three are collinear. It is easy to show that if $k=5$ the lemma is true. An induction does the rest.

Lemma 9. If $\mathbf{S} \subseteq \mathbb{R}^{2}$ is not collinear, but each of its Sylvester lines goes through point $P$, then $\mathbf{S}=\mathbf{S}_{1} \cup\{P\}$, where $\mathbf{S}_{1}$ is a collinear set and $P$ is not on the line containing $\mathbf{S}_{1}$.

Proof. Let $\mathbf{S}_{1}=\mathbf{S}-\{P\}$ and $A \in \mathbf{S}_{1}$. The lines determined by the finite set $\mathbf{S}-\{A\}$ cut the projective plane into polygonal cells. Following Kelly and Moser [10] we call those lines determined by $S-\{A\}$ which contain the edges of the polygonal cell holding $A$ the neighbors of $A$.

Case $1(P \in \mathbf{S})$. Suppose that $P A$ were not a Sylvester line in $\mathbf{S}$. Then, since all Sylvester lines of $\mathbf{S}$ pass through $P$, no line through $A$ could be a Sylvester line of $\mathbf{S}$. By Theorem 3.1 of [10], every neighbor of $A$ would then be a Sylvester line of $\mathbf{S}$; that is, every neighbor of $A$ would go through $P$ so that $A$ would have at most two neighbors and, by Theorem 2.2 of [10], our lemma would be true. We may assume, then, for each $A \in S_{1}$ the line $P A$ is a Sylvester line of $\mathbf{S}$. 
It follows that $\mathbf{S}_{1}$ must be collinear, for it it were not it would have to have a Sylvester line $A B, A, B \in S_{1}$, which either is, or is not, a Sylvester line of $\mathbf{S}$. If $A B$ is a Sylvester line of $\mathbf{S}$ it must pass through $P$ and, thereby, contain three distinct points of $\mathbf{S}$; a contradiction. But if $A B$ is not a Sylvester line of $\mathbf{S}$, then it must contain at least three points of $S$, only two of which $(A$ and $B$ ) can lie in $S_{1}$; that is to say $A B$ must contain $P$. But in that case the line $P A$ is not a Sylvester line of $\mathbf{S}$. Another contradiction. It follows that $\mathbf{S}_{\mathbf{1}}$ is collinear and the lemma is proved in this case.

Case $2(P \notin \mathbf{S})$. This is impossible, for it it were true, then the set $\mathbf{S}_{2}=\mathbf{S} \cup\{P\}$ would satisfy the conditions of Case 1 and we should have to conclude that the set $\mathbf{S}$, contrary to hypothesis, was collinear.

We now state the main result.

Theorem. If $\mathbf{S} \subseteq \mathbb{R}^{2}$ is a set of points not all on the same conic, then there is a conic containing exactly five points of $\mathbf{S}$ and determined by those five points.

Proof. Since $\mathbf{S}$ is not collinear it has Sylvester lines. Let $X, Y \in \mathbf{S}$ determine a Sylvester line. Consider the pencil, $\mathscr{P}$, of lines in $\mathbb{R}^{5}$ joining $T(Y)$ to all other points of $T(\mathbf{S})$. By Lemma $1 \operatorname{dim} T(\mathbf{S})=5$. Suppose $\mathbb{P}^{\prime}$ is a hyperplane in $\mathbb{R}^{5}$ which does not contain $T(Y)$ and which is not parallel to any of the lines in the pencil.

Let $\mathbf{S}^{\prime}=\left\{\boldsymbol{A}^{\prime} \in \mathbb{P}^{\prime} \mid \exists p \in \mathscr{P}\right.$ such that $\left.\boldsymbol{A}^{\prime}=\mathbb{P}^{\prime} \cap p\right\}$. By Lemma 3 the map $A \rightarrow A^{\prime}$, for $A \in \mathbf{S}-\{Y\}$, is $1-1$.

Suppose we could find points $A, B \in S-\{X, Y\}$ such that the points $A^{\prime}, B^{\prime}$, $X^{\prime}$ in $\mathbb{P}^{\prime}$ were collinear. Then the lines $T(Y) T(A), T(Y) T(B), T(Y) T(X)$ would be coplanar and $\{T(Y), T(X), T(A), T(B)\}$ would be a coplanar set, i.e., $\operatorname{dim}\{T(Y), T(X), T(A), T(B)\}=2$. Lemma 6 would then tell us that $\{Y, X, A, B\}$ is collinear, which is false ( $X$ and $Y$ determine a Sylvester line). Therefore no such points $A$ and $B$ exist and $X^{\prime}$ is not on the same line (in $\mathbb{P}^{\prime}$ ) with any two other points of $\mathbf{S}^{\prime}$.

It follows that in the 4-space $\mathbb{P}^{\prime}$ we can form the pencil $\mathscr{P}^{\prime}=$ $\left\{X^{\prime} A^{\prime} \mid A \in S-\{X, Y\}\right\}$ and these lines will all be distinct. We repeat the projection operation. Let $\mathbb{P}^{\prime \prime}$ be a hyperplane (a 3-space) in $\mathbb{P}^{\prime}$ which does not contain $X^{\prime}$ and is not parallel to any $X^{\prime} A^{\prime}$ in the pencil $\mathscr{P}^{\prime}$. For each $A \in \mathbf{S}-\{X, Y\}$ let $A^{\prime \prime}=X^{\prime} A^{\prime} \cap \mathbb{P}^{\prime \prime}$; we have just seen that the map $A \rightarrow A^{\prime \prime}$ is 1-1 for $A \in \mathbf{S}-\{X, Y\}$.

Let $\mathbf{S}^{\prime \prime}=\left\{A^{\prime \prime} \mid A \in \mathbf{S}-\{X, Y\}\right\} \subseteq \mathbb{R}^{3}$. Suppose that $\mathbf{S}^{\prime \prime}$ is 2-elementary (Definition $5)$, that is, $\mathbf{S}^{\prime \prime}$ determines a plane in $\mathbb{R}^{3}$ which contains exactly three points of $\mathbf{S}^{\prime \prime}$. Let $\boldsymbol{A}^{\prime \prime}, \boldsymbol{B}^{\prime \prime}, \boldsymbol{C}^{\prime \prime}$ be three such points in $\mathbf{S}^{\prime \prime}$. Then $\operatorname{dim}\left\{\boldsymbol{A}^{\prime \prime}, \boldsymbol{B}^{\prime \prime}, \boldsymbol{C}^{\prime \prime}\right\}=2$, so $\operatorname{dim}\left\{X^{\prime}, A^{\prime}, B^{\prime}, C^{\prime}\right\}=3$, and no other points of $S^{\prime}$ are in $\left\langle X^{\prime}, A^{\prime}, B^{\prime}, C^{\prime}\right\rangle$. Another pullback gives

$$
\operatorname{dim}\{T(Y), T(X), T(A), T(B), T(C)\}=4
$$

(Note that pullbacks increase the dimension by 1 and introduce exactly one new point.) By Lemma $2\{Y, X, A, B, C\}$ determines a conic in $\mathbb{R}^{2}$ which contains no 
other points of $\mathbf{S}$. This shows that if $\mathbf{S}^{\prime \prime}$ is 2-elementary then $\mathbf{S}$ is 2-elementary (Definition 3) and the theorem is true.

We assume, then, that $S^{\prime \prime}$ is not 2-elementary; that every plane in $\mathbb{R}^{3}$ determined by $\mathbf{S}^{\prime \prime}$ contains at least four points of $\mathbf{S}^{\prime \prime}$. (Of course, $\mathbf{S}^{\prime \prime}$ is not coplanar, for if it were $S^{\prime}$ would have dimension 3 and $T(S)$ would have dimension 4 .)

Motzkin [11] proved that $\mathbf{S}^{\prime \prime}$ is 2-ordinary, i.e., $\mathbf{S}^{\prime \prime}$ determines a plane whose intersection with $\mathbf{S}^{\prime \prime}$ consists of a special point $W^{\prime \prime}$ and a set of collinear points $P_{1}^{\prime \prime}, P_{2}^{\prime \prime}, \ldots, P_{r}^{\prime \prime}$ which lie on a line not containing $W^{\prime \prime}$. Note that $r \geq 3$ because every plane determined by $\mathbf{S}^{\prime \prime}$ contains at least four points.

As before

$$
\begin{array}{r}
\operatorname{dim}\left\{W^{\prime \prime}, P_{1}^{\prime \prime}, P_{2}^{\prime \prime}, \ldots, P_{r}^{\prime \prime}\right\}=2 \\
\Rightarrow \operatorname{dim}\left\{X^{\prime}, W^{\prime}, P_{1}^{\prime}, P_{2}^{\prime}, \ldots, P_{r}^{\prime}\right\}=3 \\
\Rightarrow \operatorname{dim}\left\{T(Y), T(X), T(W), T\left(P_{1}\right), T\left(P_{2}\right), \ldots, T\left(P_{r}\right)\right\}=4
\end{array}
$$

Also,

so

$$
\begin{aligned}
\operatorname{dim}\left\{P_{1}^{\prime}, P_{2}^{\prime}, \ldots, P_{r}^{\prime}\right\} & =1, \\
\operatorname{dim}\left\{X^{\prime}, P_{1}^{\prime}, P_{2}^{\prime}, \ldots, P_{r}^{\prime}\right\} & =2
\end{aligned}
$$

and

$$
\operatorname{dim}\left\{T(Y), T(X), T\left(P_{1}\right), T\left(P_{2}\right), \ldots, T\left(P_{r}\right)\right\}=3 \text {. }
$$

The last set has $r+2 \geq 5$ elements. By Lemma $8\left\{Y, X, P_{1}, P_{2}, \ldots, P_{r}\right\}$ consists of $r+1$ collinear points and one other point not on the line. Since $X Y$ is a Sylvester line we may, without loss of generality, assume that $\left\{X, P_{1}, P_{2}, \ldots, P_{r}\right\}$ is a collinear set of points, on line $a$ in $\mathbb{R}^{2}$, and $Y$ is not on $a$.

We note that if $W \in a$, then, by Lemma 4 ,

$$
\operatorname{dim}\left\{T(W), T(X), T\left(P_{1}\right), T\left(P_{2}\right), \ldots, T\left(P_{r}\right)\right\}=2
$$

and

$$
\operatorname{dim}\left\{T(Y), T(W), T(X), T\left(P_{1}\right), T\left(P_{2}\right), \ldots, T\left(P_{r}\right)\right\}=3
$$

But we have already seen that this dimension is 4 , so $W \notin a$. We now have points $X, P_{1}, P_{2}, \ldots, P_{r}$ on line $a$, points $Y$ and $W$ not on $a$; it follows that $\left\{Y, W, X, P_{1}, P_{2}, \ldots, P_{r}\right\}$ determines a unique conic $\ell$. Moreover, $\ell \cap \mathrm{S}=$ $\left\{Y, W, X, P_{1}, P_{2}, \ldots, P_{r}\right\}$, for suppose $A \in \mathscr{C} \cap \mathbf{S}$, then $T(A)$ belongs to the hyperplane

$$
\left\langle T(W), T(Y), T(X), T\left(P_{1}\right), T\left(P_{2}\right), \ldots, T\left(P_{r}\right)\right\rangle
$$

Now, either $A=Y$ or, if $A \neq Y$, we must, by Lemma 2, find $A^{\prime}$ in the 3 -flat $\left\langle W^{\prime}, X^{\prime}, P_{1}^{\prime}, P_{2}^{\prime}, \ldots, P_{r}^{\prime}\right\rangle$. Again, either $A=X$, or, if $A \neq X, A^{\prime \prime}$ belongs to the 2-flat $\left\langle W^{\prime \prime}, P_{1}^{\prime \prime}, P_{2}^{\prime \prime}, \ldots, P_{r}^{\prime \prime}\right\rangle$. Therefore $A^{\prime \prime}=W^{\prime \prime}$ or $A^{\prime \prime}=P_{i}^{\prime \prime}$ for some $i$. Since all the maps are 1-1 this means that $A=W$ or $A=P_{i}$ for some $i$. 
Our situation now is this: having started with $X, Y \in \mathrm{S}, X Y$ a Sylvester line, we have found points $W, P_{1}, \ldots, P_{r}$ in $\mathrm{S}$ such that

(i) $\left\langle W^{\prime \prime}, P_{1}^{\prime \prime}, \ldots, P_{r}^{\prime \prime}\right\rangle$ is an ordinary plane in $\mathbb{R}^{3}$;

(ii) there is a line, $a$, in $\mathbb{R}^{2}$ such that

$$
a \cap \mathbf{S}=\left\{X, P_{1}, \ldots, P_{r}\right\}
$$

(iii) $\left\{X, Y, W, P_{1}, \ldots, P_{r}\right\}$ determines a conic $\mathscr{C}=a \cup W Y$ which contains no other points of $S$.

Suppose the line $W Y$ is not a Sylvester line of $\mathbf{S}$. Then, since $\ell \cap \mathbf{S}=$ $\left\{X, Y, W, P_{1}, \ldots, P_{r}\right\}, W Y \cap S=\left\{W, Y, P_{j}\right\}$ for some $j$; but then $W Y$ is a line containing exactly three points of $\mathrm{S}$, so by Lemma $7 \mathrm{~S}$ is 2-elementary and the theorem is proved. We may assume, then, that $W Y$ is a Sylvester line of $\mathbf{S}$.

We return, now, to the three-dimensional hyperplane $\mathbb{P}^{\prime \prime}$. Suppose that $W^{\prime \prime} A^{\prime \prime}=$ $W^{\prime \prime} B^{\prime \prime}$ implied $A=B$ for $A, B \in \mathbf{S}-\{X, Y, W\}$; i.e., $W^{\prime \prime}$ is not collinear with any other two points of $S^{\prime \prime}$. Form the pencil of lines $W^{\prime \prime} A^{\prime \prime}, A^{\prime \prime} \in \mathbf{S}^{\prime \prime}-\left\{W^{\prime \prime}\right\}$, and cut the pencil with a 2 -flat $\mathbb{P}^{\prime \prime \prime}$ which does not contain $W^{\prime \prime}$ and is not parallel to any of the lines in the pencil. Define

$$
\mathbf{S}^{\prime \prime \prime}=\left\{A^{\prime \prime \prime} \mid A^{\prime \prime}=W^{\prime \prime} A^{\prime \prime} \cap \mathbb{P}^{\prime \prime} \text { for } A \text { in } S-\{X, Y, W\}\right\} \text {. }
$$

If the points of $S^{\prime \prime \prime}$ were collinear the points of $S^{\prime \prime}$ would be coplanar, those of $\mathbf{S}^{\prime}$ would lie in a 3 -flat, and those of $T(\mathbf{S})$ in a 4-flat; that is, $\mathbf{S}$ would be a co-conic set. That is untrue, so $\mathbf{S}^{\prime \prime \prime}$ is not a collinear set and must have Sylvester lines. Let $A, B \in \mathbf{S}-\{X, Y, W\}$ be points of $\mathbf{S}$ which give rise to a Sylvester line $A^{\prime \prime \prime} B^{\prime \prime \prime}$ for the set $\mathbf{S}^{\prime \prime \prime}$.

It follows that if $W^{\prime \prime}$ is not collinear with any other two points of $\mathbf{S}^{\prime \prime}$, then $\left\langle W^{\prime \prime}, A^{\prime \prime}, B^{\prime \prime}\right\rangle$ is an elementary 2-flat in $\mathbf{S}^{\prime \prime}$ (that is, it contains only those three points of $\left.\mathbf{S}^{\prime \prime}\right)$, so $\left\langle X^{\prime}, W^{\prime}, A^{\prime}, B^{\prime}\right\rangle$ is an elementary 3-flat in $\mathbf{S}^{\prime}$, and $\langle T(Y), T(X), T(W), T(A), T(B)\rangle$ is an elementary 4-flat in $\mathbb{R}^{5}$. In other words, if $W^{\prime \prime}$ is not collinear with two other points of $\mathbf{S}^{\prime \prime}$ the theorem is true.

We assume, then, that $W^{\prime \prime}$ lies in a line containing at least two other points of $\mathbf{S}^{\prime \prime}$. Let $b$ be such a line and let

$$
b \cap \mathbf{S}^{\prime \prime}=\left\{W^{\prime \prime}, Q_{1}^{\prime \prime}, \ldots, Q_{s}^{\prime \prime}\right\}, \quad s \geq 2 .
$$

Then

so

$$
\operatorname{dim}\left\{W^{\prime \prime}, Q_{1}^{\prime \prime}, \ldots, Q_{s}^{\prime \prime}\right\}=1,
$$$$
\operatorname{dim}\left\{X^{\prime}, W^{\prime}, Q_{1}^{\prime}, \ldots, Q_{s}^{\prime}\right\}=2
$$

and

$$
\operatorname{dim}\left\{T(Y), T(X), T(W), T\left(Q_{1}\right), \ldots, T\left(Q_{s}\right)\right\}=3 \text {. }
$$

By Lemma $8\left\{Y, X, W, Q_{1}, \ldots, Q_{s}\right\}$ consist of $s+2$ collinear points and one other point not on the line. Since $X Y$ and $W Y$ are Sylvester lines and $W$ is collinear with the points $Q_{i}$ we must have the picture shown in Fig. 2 (the points on the line may, of course, appear in any order). 


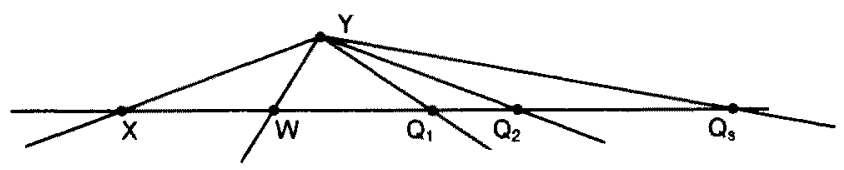

Fig. 2

It is important to note that the line $b$ is unique-it is the only line containing $W^{\prime \prime}$ and at least two other points of $\mathbf{S}^{\prime \prime}$, for if $c$ were another such line the pullback argument above would still lead to the line $X W$ pictured above.

We have reduced the problem to this: $S^{\prime \prime}$ determines an ordinary plane, $\left\langle W^{\prime \prime}, P_{1}^{\prime \prime}, \ldots, P_{r}^{\prime \prime}\right\rangle$, in which $W^{\prime \prime}$ is the special point. There is exactly one line, $b$, determined by $\mathbf{S}^{\prime \prime}$ which contains $W^{\prime \prime}$ and at least two other points of $\mathbf{S}^{\prime \prime}$ :

$$
b \cap \mathbf{S}^{\prime \prime}=\left\{W^{\prime \prime}, Q_{1}^{\prime \prime}, \ldots, Q_{s}^{\prime \prime}\right\}, \quad s \geq 2
$$

There may be other points of $S^{\prime \prime}$ in $\mathbb{R}^{3}$, but we can see at least the ones shown in Fig. 3.

Notice that $Q_{i}^{\prime \prime \prime}=Q_{j}^{\prime \prime \prime}$ (in $\mathbf{S}^{\prime \prime \prime}$ ) for all $i$ and $j$. Call this common point $Q^{\prime \prime \prime}$, i.e., $Q^{\prime \prime}=Q_{i}^{\prime \prime \prime}$ for all $i$. Notice also that except for this point $Q^{\prime \prime \prime}$ the projection is $1-1$.

$\mathbf{S}^{\prime \prime \prime}$ is not collinear. Suppose that $\mathbf{S}^{\prime \prime \prime}$ has a Sylvester line $A^{\prime \prime \prime} B^{\prime \prime \prime}$ which does not go through $Q^{\prime \prime \prime}$. Then $\left\langle W^{\prime \prime}, A^{\prime \prime}, B^{\prime \prime}\right\rangle$ is an elementary 2 -flat, $\left\langle X^{\prime}, W^{\prime}, A^{\prime}, B^{\prime}\right\rangle$ is an elementary 3-flat, and $\langle T(Y), T(X), T(W), T(A), T(B)\rangle$ is an elementary 4-flat. In other words, if $\mathbf{S}^{\prime \prime \prime}$ has a Sylvester line which does not go through $Q^{\prime \prime \prime}$, then the theorem is true.

So we may assume that all the Sylvester lines of $\mathbf{S}^{\prime \prime \prime}$ go through the single point $Q^{\prime \prime \prime}$. By Lemma $9 \mathbf{S}^{\prime \prime \prime}$ consists of $Q^{\prime \prime \prime}$ and a set of collinear points which lie on a line not containing $Q^{\prime \prime \prime}$. It follows that all points of $\mathbf{S}^{\prime \prime}$ except for $Q_{1}^{\prime \prime}, Q_{2}^{\prime \prime}, \ldots, Q_{s}^{\prime \prime}$ lie in the same plane as do $P_{1}^{\prime \prime}, P_{2}^{\prime \prime}, \ldots, P_{r}^{\prime \prime}$ and $W^{\prime \prime}$.

Next, consider the set $\mathbf{S}^{\prime \prime}-\left\{W^{\prime \prime}, Q_{1}^{\prime \prime}, Q_{2}^{\prime \prime}, \ldots, Q_{s}^{\prime \prime}\right\}$. If this set is not collinear, then we can find points $A^{\prime \prime}$ and $B^{\prime \prime}$ in it which determine a Sylvester line, $d$. If

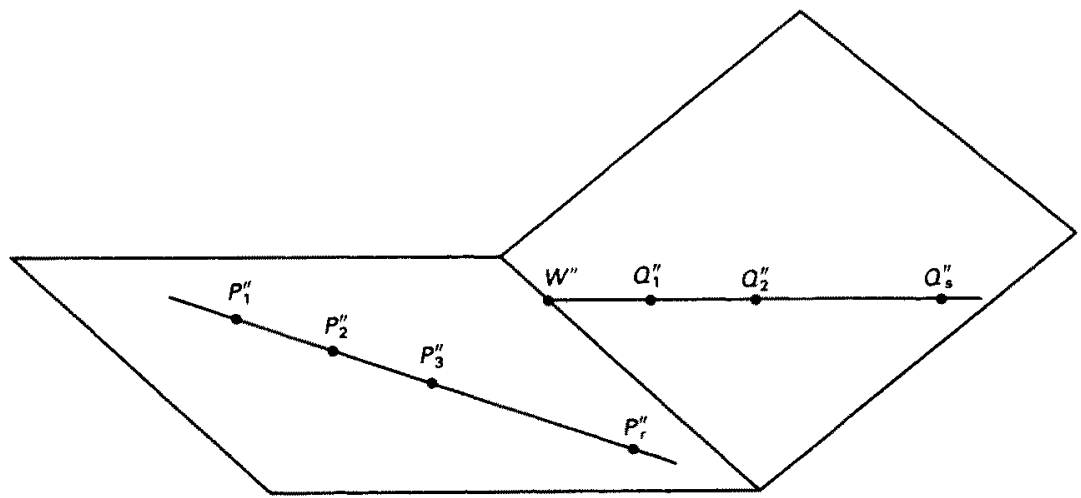

Fig. 3 


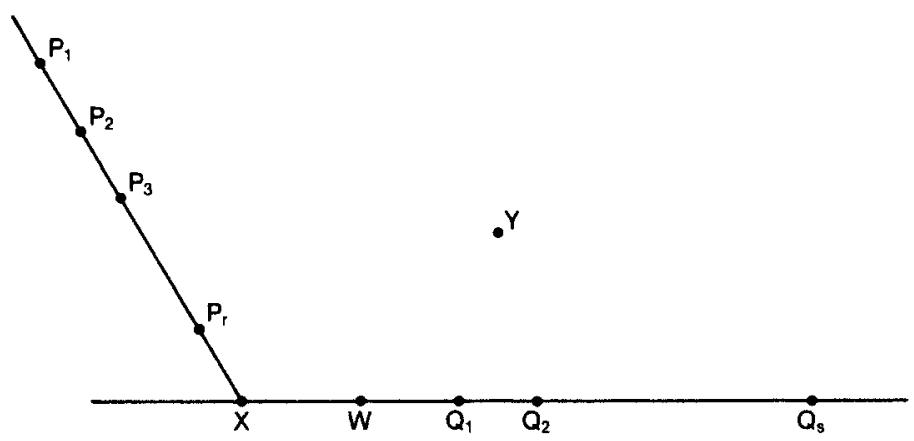

Fig. 4

all the Sylvester lines for this set went through $W^{\prime \prime}$ then, by Lemma 9 , the set would be collinear, so we may assume that $d$ does not contain $W^{\prime \prime}$. It follows that $\left\{\boldsymbol{A}^{\prime \prime}, \boldsymbol{B}^{\prime \prime}, Q_{1}^{\prime \prime}\right\}$ determines a plane which contains exactly three points of $\mathbf{S}^{\prime \prime}$. We have already seen that in this case $\mathbf{S}$ is 2-elementary and the theorem is true.

We may assume, then, that the points of $\mathbf{S}^{\prime \prime}-\left\{W^{\prime \prime}, Q_{1}^{\prime \prime}, Q_{2}^{\prime \prime}, \ldots, Q_{4}^{\prime \prime}\right\}$ are collinear. Therefore

$$
\mathbf{S}^{\prime \prime}=\left\{W^{\prime \prime}, Q_{1}^{\prime \prime}, Q_{2}^{\prime \prime}, \ldots, Q_{s}^{\prime \prime}, P_{1}^{\prime \prime}, P_{2}^{\prime \prime}, \ldots, P_{r}^{\prime \prime}\right\}
$$

In other words, $\mathbf{S}^{\prime \prime}$ lies in the two skew lines $a$ and $b$. Doing a pullback similar to the one done earlier, we have

so

$$
\operatorname{dim}\left\{P_{1}^{\prime \prime}, P_{2}^{\prime \prime}, \ldots, P_{r}^{\prime \prime}\right\}=1 \quad(r \geq 3),
$$

and

$$
\operatorname{dim}\left\{X^{\prime}, P_{1}^{\prime}, P_{2}^{\prime}, \ldots, P_{r}^{\prime}\right\}=2 \text {, }
$$

$$
\operatorname{dim}\left\{T(Y), T(X), T\left(P_{1}\right), T\left(P_{2}\right), \ldots, T\left(P_{r}\right)\right\}=3 \text {. }
$$

By Lemma 8 the set $\mathbf{S}$ must look like Fig. 4 (the order of the points on the lines is irrelevant). Clearly, $\left\{P_{1}, P_{2}, W, Q_{1}, Y\right\}$ determines an elementary conic, so $\mathrm{S}$ is 2-elementary, and the theorem is proved.

It seems reasonable to conjecture that every non- $n$th order configuration in the plane determines an $n$th order elementary curve in the plane. That is, given a set of points in the plane not all lying on an algebraic curve of order $n$, there must be at least one curve of order $n$ containing exactly $n(n+3) / 2$ points of the set and determined by those points.

Proof of this conjecture would add considerable interest and importance to Sylvester-Gallai theory.

\section{Acknowledgment}

We want to thank William Moser for a lucid talk in Toronto at an AMS special session on geometry; it was our introduction to the Sylvester problem. Our thanks also to two referees for their encouraging words and helpful criticisms. 


\section{References}

1. W. E. Bonnice and L. M. Kelly, On the number of ordinary planes, J. Combin. Theory Ser. A 11 (1971), 45-53.

2. P. B. Borwein, On Sylvester's problem and Haar spaces, Pacific J. Math. 109 (1983), 275-278.

3. H. M. S. Coxeter, The Real Projective Plane, 2nd edn., Cambridge University Press, Cambridge, 1955.

4. H. M. S. Coxeter, Introduction to Geometry, Wiley, New York, 1961. Footnote, p. 65.

5. P. Erdös, Problem \#4065, Amer. Math. Monthly 50 (1943), 65.

6. S. Hansen, A generalization of a theorem of Sylvester on the lines determined by a finite point set, Math. Scand. 16 (1965), 175-180.

7. S. Hansen, Contributions to the Sylvester-Gallai Theory, Polyteknisk Forlag, Copenhagen, 1981.

8. R. Hartshorne, Algebraic Geometry, Springer-Verlag, New York, 1977.

9. L. M. Kelly, Classroom notes, Amer. Math. Monthly 55 (1948), 28.

10. L. M. Kelly and W. O. J. Moser, On the number of ordinary lines determined by $n$ points, Canad. J. Math. 10 (1958), 210-219.

11. T. Motzkin, The lines and planes connecting the points of a finite set, Trans. Amer. Math. Soc. 70 (1951), 451-464.

12. R. Steinberg, Solution to problem \#4065, Amer. Math. Monthly 51 (1944), 169-171.

13. J. J. Sylvester, Mathematical questions and solutions, 11851, The Educatonal Times (1983), 98.

Received March 6, 1986, and in revised form November 10, 1986. 